\title{
LETTER
}

\section{Mass Spectrometry-Based Proteomics to Understand Schizophrenia}

\author{
Daniel Martins-de-Souza ${ }^{1,2,3,4}$ iD \\ ${ }^{1}$ Laboratory of Neuroproteomics, Department of Biochemistry and Tissue Biology, Institute of Biology, University \\ of Campinas, Campinas, SP, Brazil \\ ${ }^{2}$ Experimental Medicine Research Cluster (EMRC), University of Campinas, Campinas, 13083-862, SP, Brazil \\ ${ }^{3}$ National Institute of Biomarkers in Neuropsychiatry, National Council for Scientific and Technological \\ Development, São Paulo, SP, Brazil \\ ${ }^{4}$ D'Or Institute for Research and Education (IDOR), São Paulo, SP, Brazil
}

Psychiatric disorders are the most disabling disorders of humankind. From an economic and social point of view, a person affected by these diseases may lose about 30 years of their lives in an unproductive manner [1]. Although psychiatric patients have been diagnosed and treated for more than a century, these diseases are incurable, and the current medications only partially alleviate symptoms. These hurdles are mostly because we do not understand the biology behind these diseases. Understanding the most elementary molecular processes involved in the development and establishment of these multifactorial diseases is mandatory for better treating them, which will in turn improve patients' lives.

Proteomics emerged in the post genomic era as an alternative toolbox that, by definition, is appropriate to study multifactorial diseases such as psychiatric disorders. In the case of schizophrenia, we can easily see that proteomics has opened roads that have been traveled by scientists in the last decade.

Around 20 years ago, the first proteomic study approaching schizophrenia was published, combining two-dimensional gel electrophoresis and mass spectrometry [2]. From then on, proteomic methods have evolved towards automated liquid chromatography coupled to high-resolution tandem mass spectrometry (LC-MS/MS) and its derivations in qualitative and quantitative terms. The whole proteomic toolbox for LC-MS/MS-based shotgun quantitative proteomics has been employed to decipher the pathobiology of schizophrenia from the molecular point of view: 1DLC or 2DLC in terms of liquid chromatography (offline and online); data-dependent analysis (DDA) and data-independent analysis (DIA) - even including ion mobility - in terms of mass spectrometry [3]. In quantitative terms, different stable isotope labeling techniques - e.g., Isotope-Coded Protein Labeling (ICPL), Isobaric tag for relative and absolute quantitation (iTRAQ) - and label-free approaches (spectral counting and $\mathrm{MS}^{\mathrm{E}}$ ) have also been used [4]. Finally, targeted proteomics (selected reaction monitoring, SRM) have also been employed $[5,6]$. These technologies were employed mostly to study postmortem brains and blood plasma or serum. However, other human organs such as the skin and liver were also explored as well as several in vivo and in vitro models.

While studying the schizophrenia brains, the most consistent differences observed in the proteomes were those associated with energy metabolism, myelination, cytoskeleton assembly, alternative splicing (mRNA processing), and synaptic transmission. Differences associated with synaptic deficits have largely been documented, not only by proteomic analysis but also by large genomic studies. Some of the most commonly found genes or proteins associated to schizophrenia with synaptic function, according to the UniProt, are depicted in Figure 1.

Cite: Martins-de-Souza, D. Mass Spectrometry-Based Proteomics to Understand Schizophrenia. Braz. J. Anal. Chem., 2020, 7 (29), pp 13-17. doi: http://dx.doi.org/10.30744/brjac.2179-3425.letter-dmsouza 


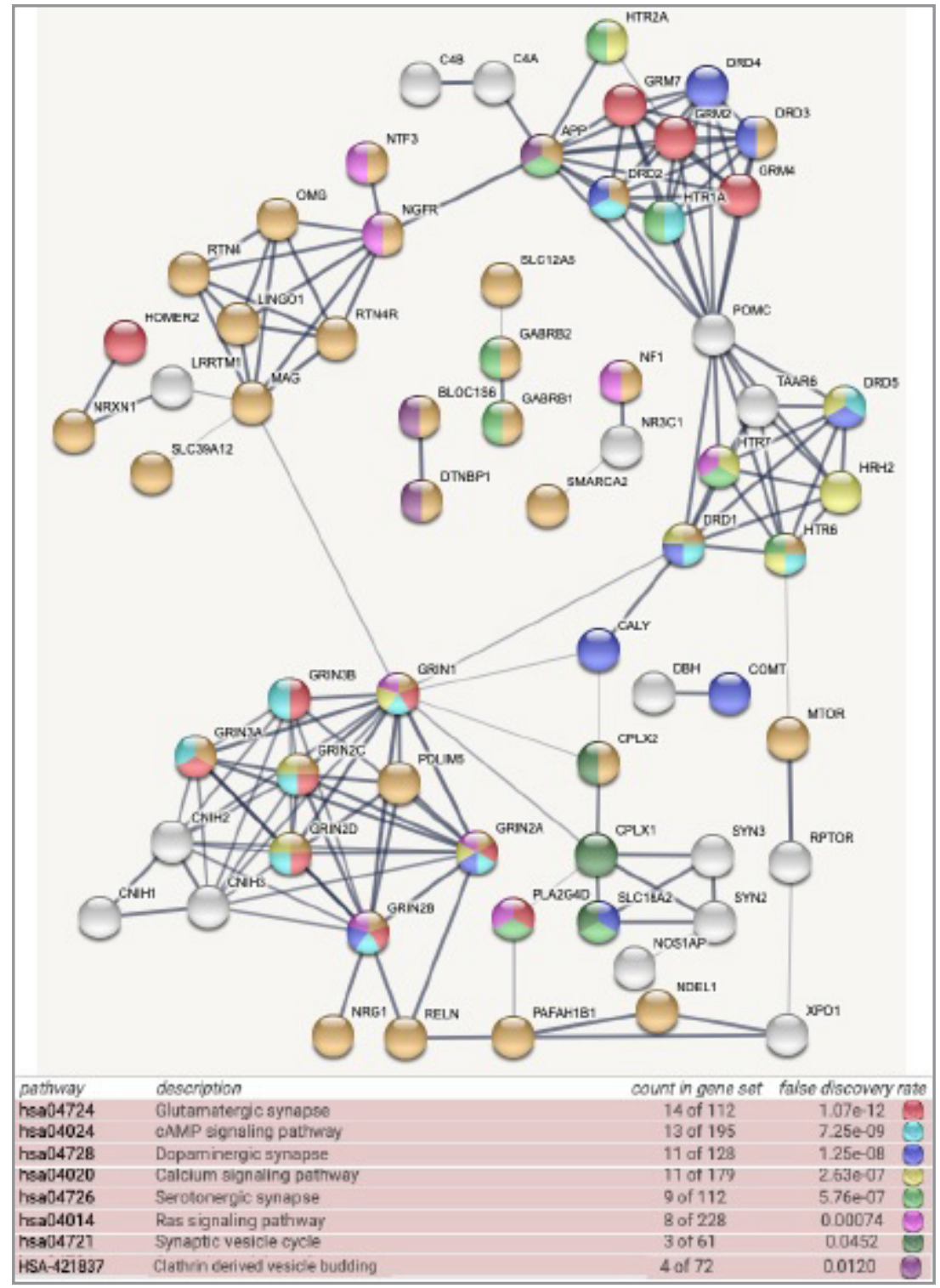

Figure 1. Genes and proteins involved in the synaptic dysfunction associated with schizophrenia, (color coded to the major biochemical pathways they are involved in). Dopamine, serotonin, and glutamate receptors are represented majorly. In silico analysis performed in STRING [14].

Interestingly, proteomic alterations observed in postmortem schizophrenia brains have also been observed in induced pluripotent stem cell (iPSC)-derived cerebral organoids generated from the cells of schizophrenia patients (not published). Since proteomics have highlighted the most altered biological processes in schizophrenia, these need to be studied more deeply.

Using in vivo and in vitro pre-clinical models, we were able to prove that the energy-metabolismassociated differences observed in schizophrenia brains are likely to happen in oligodendrocytes, which are the cells that produce myelin [7]. In Figure 2, based on data from the literature, we can see how the main energy metabolism proteins are associated with classical myelination markers. More specifically, our results indicate that glycolysis seems to be essential in this regard, which is also supported by the analysis conducted in Figure 2. Oligodendrocytes became one of the main topics of our studies since we believe that schizophrenia is not only a neuronal disease, as it has been treated so far, but it can also be a disease 
centered on glia cells [8]. It is known that current antipsychotic medication mostly affects the function of neurons. We have also been investigating whether antipsychotics target oligodendrocytes [9]. By realizing that this does happen, we have also been investigating alternative treatments, such as those associated with the endocannabinoid system, as a means of better treating schizophrenia [10].

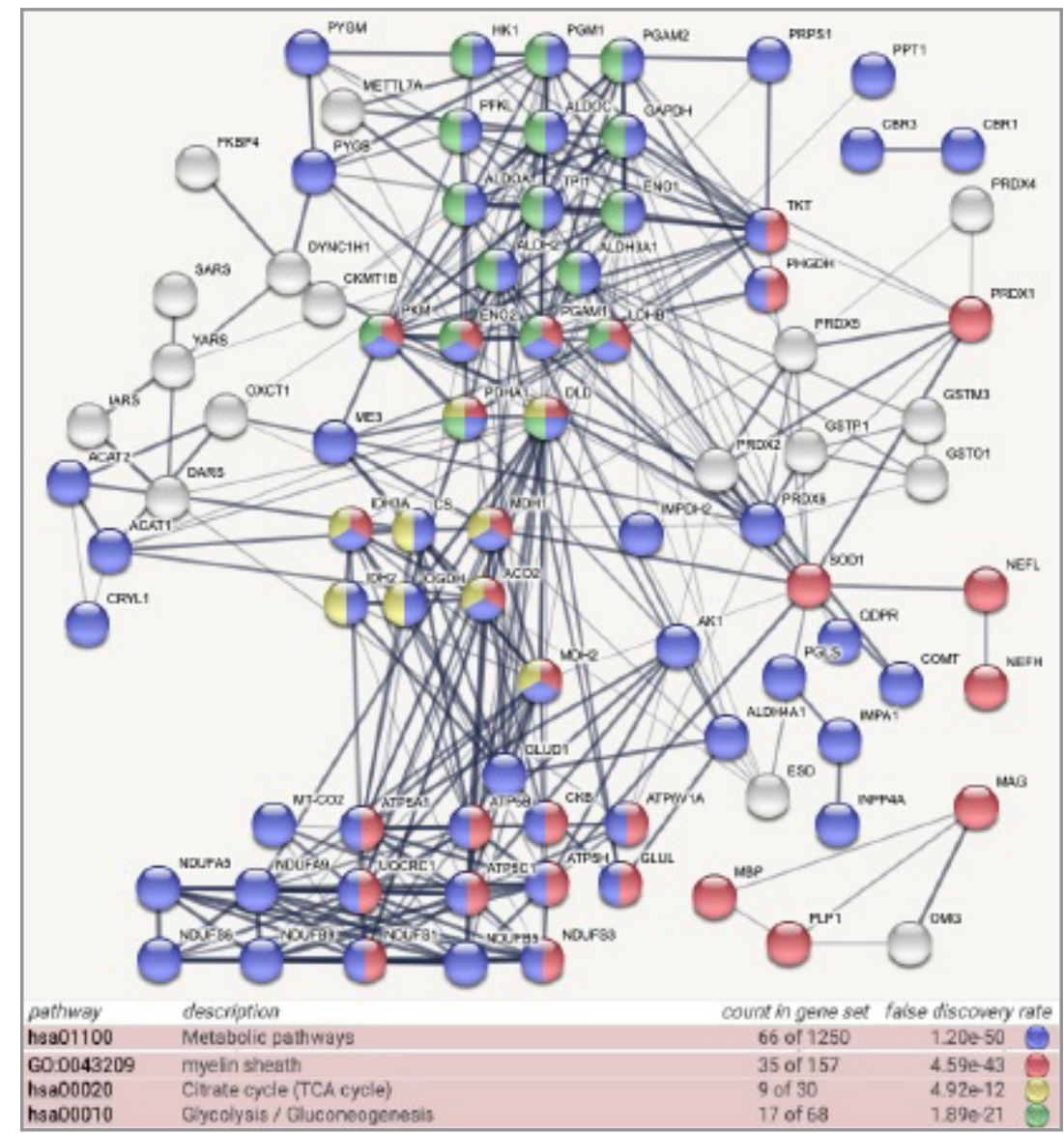

Figure 2. Differentially expressed proteins in schizophrenia samples associated with energy metabolism and myelination. Their strong connectivity shows their direct relation, as we demonstrated experimentally. The central role of glycolysis is also highlighted. In silico analysis perfomed in STRING [14].

In addition, more recently we have been searching for protein biomarkers that could predict an unsuccessful response to antipsychotics in the blood serum or plasma of schizophrenia patients. This is important because almost half of schizophrenia patients do not respond properly to the first round of medication. When medication does not function properly, the disease severity increases, and patients never recover their full brain performance. Moreover, given the side effects of antipsychotic medications, there has been a significant drop in medication usage by patients, which can only worsen the symptoms and, therefore, the mental health of the patients. Thus far, we were able to generate panels of lipid [11] and protein biomarkers [12], which may be implemented as biochemical tests for the prediction of a successful drug response. Proteomic signatures have also been used to build a diagnostic test for schizophrenia [13], which was even commercialized, but later discontinued.

In the last two decades, proteomics has added significant value to the understanding of schizophrenia, which is also true for the other psychiatric disorders. These investigations will end up improving the lives of patients since the next generation of medication may be based on the molecular underpinnings associated 
with the disease. Eventually, we may also have biomarker tests in the future for better diagnosis and treatment outcomes from proteomic investigations.

\section{Acknowledgments}

We dedicate our work to psychiatric patients and their families. Our research was funded by FAPESP (São Paulo Research Foundation), grant 2017/25588-1, and CNPq (The Brazilian National Council for Scientific and Technological Development).

\section{REFERENCES}

1. Insel, T. 2013. https://www.youtube.com/watch?v=u4m65sbqbhY

2. Johnston-Wilson, N. L.; Sims, C. D.; Hofmann, J. P.; Anderson, L.; Shore, A. D.; Torrey, E. F.; Yolken, R. H. Mol Psychiatry, 2000, 5 (2), pp 142-149 (https://doi.org/10.1038/sj.mp.4000696).

3. Souza G. H.; Guest, P. C.; Martins-de-Souza, D. Methods Mol Biol., 2017, 1546, pp 57-73 (https://doi. org/10.1007/978-1-4939-6730-8_4).

4. Smith, B. J.; Martins-de-Souza, D.; Fioramonte, M. Methods Mol Biol., 2019, 1916, pp 3-39 (https://doi. org/10.1007/978-1-4939-8994-2_1).

5. Martins-de-Souza, D.; Alsaif, M.; Ernst, A.; Harris, L. W.; Aerts, N.; Lenaerts, I.; Peeters, P. J.; Amess, B.; Rahmoune, H.; Bahn, S.; Guest, P. C. BMC Res Notes, 2012, 5 (146) (https://doi.org/10.1186/17560500-5-146).

6. Velásquez, E.; Martins-de-Souza, D.; Velásquez, I.; Carneiro, G. R. A.; Schmitt, A.; Falkai, P.; Domont, G. B.; Nogueira, F. C. S. J Proteome Res., 2019, 18 (12) pp 4240-4253 (https://doi.org/10.1021/acs. jproteome.9b00398)

7. Guest, P. C.; Iwata, K.; Kato, T. A.; Steiner, J.; Schmitt, A.; Turck, C. W.; Martins-de-Souza, D. Front Cell Neurosci., 2015, 9, Article 180 (https://doi.org/10.3389/fncel.2015.00180).

8. Gouvêa-Junqueira, D.; Falvella, A. C. B.; Antunes, A. S. L. M.; Seabra, G.; Brandão-Teles, C.; Martins-de-Souza, D.; Crunfli, F. Front. Psychiatry, 2020, 11, Article 379 (https://doi.org/10.3389/ fpsyt.2020.00379).

9. Brandão-Teles, C.; de Almeida, V.; Cassoli, J. S.; Martins-de-Souza, D. Front. Pharmacol. 2019, 10, Article 186 (https://doi.org/10.3389/fphar.2019.00186).

10. de Almeida, V.; Martins-de-Souza, D. Eur Arch Psychiatry Clin Neurosci., 2018, 268 (7) pp 727-737 (https://doi.org/10.1007/s00406-018-0874-6).

11. Aquino, A.; Alexandrino, G. L.; Guest, P. C.; Augusto, F.; Gomes, A. F.; Murgu, M.; Steiner, J.; Martinsde-Souza, D. Front. Psychiatry., 2018, 9, Article 209 (https://doi.org/10.3389/fpsyt.2018.00209).

12. Garcia-Rosa, S.; Carvalho, B. S.; Guest, P. C.; Steiner, J.; Martins-de-Souza, D. J Proteomics, 2020, 224, 103813 (https://doi.org/10.1016/j.jprot.2020.103813).

13. Schwarz, E.; Guest, P. C.; Rahmoune, H.; Harris, L. W.; Wang, L.; Leweke, F. M.; Rothermundt, M.; Bogerts, B.; Koethe, D.; Kranaster, L.; et al. Mol Psychiatry., 2012, 17 (5) pp 494-502 (https://doi. org/10.1038/mp.2011.42).

14. Szklarczyk, D.; Gable, A. L.; Lyon, D.; Junge, A.; Wyder, S.; Huerta-Cepas, J.; Simonovic, M.; Doncheva, N. T.; Morris, J. H.; Bork, P.; et al. Nucleic Acids Res., 2019, 47 (D1) D607-D613 (https:// doi.org/10.1093/nar/gky1131). 


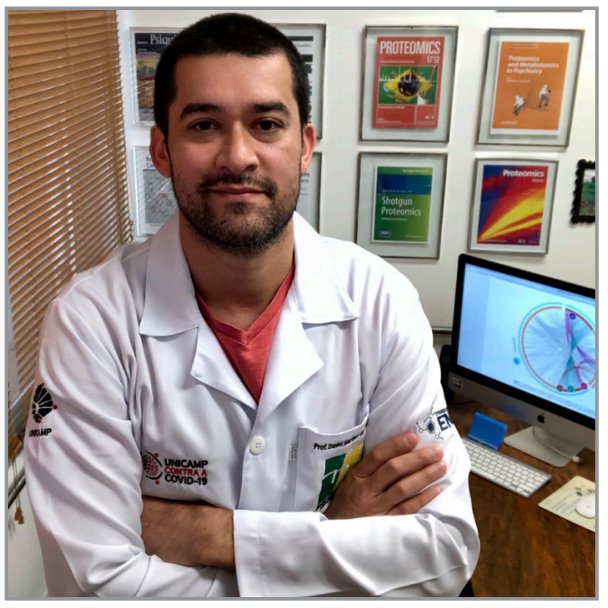

Daniel Martins-de-Souza is Associate Professor of Biochemistry, Advisor to the Vice-President for Research at the University of Campinas (Unicamp), and one of the Coordinators of the Biology Area at FAPESP (São Paulo Research Foundation). Daniel founded the Laboratory of Neuroproteomics in 2014 right after having returned from Germany after a period of two years as Principal Investigator at LMU, Ludwig Maximilians Universität Muenchen (2012-2014). His expertise is mainly in mass spectrometry-based proteomics (shotgun proteomics) and systems biology in silico, employed in clinical and pre-clinical models for psychiatric disorders. Daniel is a Biologist (2003) with a PhD in Biochemistry (2008), which was obtained at Unicamp. His postdoctoral experience was at the Max Planck Institute of Psychiatry in Germany (2008-2010) and at the University of Cambridge in the United Kingdom (20102012), where he also led the Mass Spectrometry Unit at the Cambridge Center for Neuropsychiatric Research (CCNR). At the same time, Daniel was a consultant for Psynova Neurotech Ltd. Daniel is an Affiliated Member of the Brazilian Academy of Sciences (2017-2021) and an Affiliated Member of the Academy of Sciences of the State of São Paulo (2019-2023). He is also a founding member of BrProt (Brazilian Society of Proteomics) and a board member of BrMass (Brazilian Society of Mass Spectrometry). He was elected to the board of the Human Proteome Organization (2015-2017) and has been a member of the Human Brain Proteome Project Steering Committee since 2015. Daniel is one of the Associate Editors of npj Schizophrenia (Nature), a member of the editorial board of 7 other scientific journals, and a permanent editor of a book series entitled "Proteomics, Metabolomics, Interactomics and Systems Biology" by Springer-Nature. Thus far, he has given more than 60 oral presentations at international conferences. 